\section{(6) OPEN ACCESS}

\title{
PTPN22 is associated with susceptibility to psoriatic arthritis but not psoriasis: evidence for a further PsA-specific risk locus
}

\author{
John Bowes, ${ }^{1}$ Sabine Loehr, ${ }^{2}$ Ashley Budu-Aggrey, ${ }^{1,3}$ Steffen Uebe, ${ }^{2}$ lan N Bruce, ${ }^{1,3,4}$ \\ Marie Feletar, ${ }^{5}$ Helena Marzo-Ortega, ${ }^{6}$ Philip Helliwell, ${ }^{6}$ Anthony W Ryan, ${ }^{7}$ \\ David Kane, ${ }^{8}$ Eleanor Korendowych, ${ }^{9}$ Gerd-Marie Alenius, ${ }^{10}$ Emiliano Giardina, ${ }^{11}$ \\ Jonathan Packham, ${ }^{12}$ Ross McManus, ${ }^{7}$ Oliver FitzGerald, ${ }^{13}$ Matthew A Brown, ${ }^{14}$ \\ Frank Behrens, ${ }^{15}$ Harald Burkhardt, ${ }^{15}$ Neil McHugh, ${ }^{9}$ Ulrike Huffmeier, ${ }^{2}$ Pauline Ho, ${ }^{1,4}$ \\ Andre Reis, ${ }^{2}$ Anne Barton 1,3,4
}

Handling editor Tore K Kvien

- Additional material is published online only. To view please visit the journal online (http://dx.doi.org/10.1136/ annrheumdis-2014-207187)

For numbered affiliations see end of article.

\section{Correpondence to} Professor Anne Barton, Arthritis Research UK Centre for Genetics and Genomics, The University of Manchester, Manchester M13 9PT, UK anne.barton@manchester.ac.uk

Received 19 December 2014 Revised 3 March 2015 Accepted 5 April 2015 Published Online First 28 April 2015

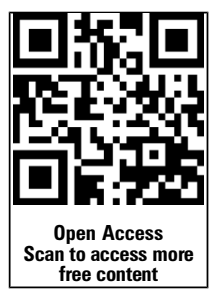

CrossMark

\section{SLinked}

- http://dx.doi.org/10.1136 annrheumdis-2014-207190

To cite: Bowes J, Loehr $S$, Budu-Aggrey $\mathrm{A}$, et al. Ann Rheum Dis 2015;74: 1882-1885.

\section{ABSTRACT}

Objectives Psoriatic arthritis (PsA) is a chronic inflammatory arthritis associated with psoriasis; it has a higher estimated genetic component than psoriasis alone, however most genetic susceptibility loci identified for PsA to date are also shared with psoriasis. Here we attempt to validate novel single nucleotide polymorphisms selected from our recent PsA Immunochip study and determine specificity to PsA. Methods A total of 15 single nucleotide polymorphisms were selected $\left(P_{\text {Immunochip }}<1 \times 10^{-4}\right)$ for validation genotyping in 1177 cases and 2155 controls using TagMan. Meta-analysis of Immunochip and validation data sets consisted of 3139 PsA cases and 11078 controls. Novel PsA susceptibility loci were compared with data from two large psoriasis studies (WTCCC2 and Immunochip) to determine PsA specificity. Results We found genome-wide significant association to rs2476601, mapping to PTPN22 ( $p=1.49 \times 10^{-9}$, $\mathrm{OR}=1.32$ ), but no evidence for association in the psoriasis cohort ( $p=0.34$ ) and the effect estimates were significantly different between PsA and psoriasis $\left(p=3.2 \times 10^{-4}\right)$. Additionally, we found genome-wide significant association to the previously reported psoriasis risk loci; NOS2 (rs4795067, $p=5.27 \times 10^{-9}$ ). Conclusions For the first time, we report genome-wide significant association of PTPN22 (rs2476601) to PsA susceptibility, but no evidence for association to psoriasis.

\section{INTRODUCTION}

Psoriatic arthritis (PsA) is a chronic inflammatory arthritis associated with psoriasis; in UK populations the prevalence rate of PsA in patients with psoriasis is estimated to be $14 \% .{ }^{1}$ While psoriasis has a serious impact on the patient's quality of life, those suffering from PsA have been found to have a lower quality of life than psoriasis alone. ${ }^{2}$

PsA is a complex disease with environmental and genetic risk factors contributing to the overall liability. The genetic factors contributing to the susceptibility of PsA are not fully understood, but PsA is estimated to have a larger genetic component than psoriasis. $^{3}$ This suggests a substantial difference in the genetic architecture of the two diseases with a heavier genetic burden for PsA. Many of the genetic risk loci reported as associated with PsA susceptibility are shared with psoriasis indicating the importance of pleiotropic effects within shared molecular pathways mediated by the presence of cutaneous psoriasis in both phenotypes. Recent studies have identified PsA-specific loci that begin to explain this increased burden; the presence of glutamic acid at the amino acid position 45 in HLA-B has been shown to be a risk factor for PsA in a psoriasis cohort and our recent Immunochip study confirmed the independent $H L A-B$ association. ${ }^{4}$ In addition, we reported evidence for a PsA-specific risk locus at chromosome $5 \mathrm{q} 31$ and distinct PsA variants at the IL23R locus.

The aim of the current study was to test the loci at suggestive levels of significance in our recent Immunochip analysis to identify novel PsA loci in a large collection of PsA cases and controls collected from the UK, Ireland, Germany, Australia, Sweden and Italy.

\section{METHODS}

\section{Samples}

All samples included in this study were of European ancestry and provided written informed consent. Summary statistics and genotype data were available from the PsA Immunochip study comprising 1962 cases and 8923 controls. $^{5}$ In addition genotype data was available for the psoriasis Wellcome Trust Case Control Consortium 2 (WTCCC2) study which contained 1784 psoriasis samples following exclusion of known PsA samples and 5175 controls. $^{6} \mathrm{~A}$ total of 1352 PsA case and 2164 control DNA samples, independent of those tested as part of the Immunochip study, were available for genotyping collected from Germany ( cases $=508$, controls $=920)$, Sweden $($ cases $=417$, controls $=1079$ ) and Italy (cases $=427$, controls=165). A description of clinical characteristics for the three cohorts is provided in online supplementary table S1. Data for a total of 3139 PsA cases and 11078 controls were available for this study following quality control.

\section{SNP selection and genotyping}

A total 15 single nucleotide polymorphisms (SNPs) were selected from the Immunochip study based on 
Table 1 Summary statistics for Immunochip, validation and meta-analysis of selected SNPs

\begin{tabular}{|c|c|c|c|c|c|c|c|c|c|c|c|c|c|}
\hline \multirow[b]{2}{*}{ rs } & \multirow[b]{2}{*}{ chr } & \multirow[b]{2}{*}{ bp } & \multirow[b]{2}{*}{ Gene } & \multirow[b]{2}{*}{ Risk/non-risk } & \multicolumn{3}{|c|}{$\begin{array}{l}\text { Immunochip } \\
\text { (cases=1962, } \\
\text { controls=8923) }\end{array}$} & \multicolumn{2}{|c|}{$\begin{array}{l}\text { Validation } \\
\text { (cases=1177, } \\
\text { controls=2155) }\end{array}$} & \multicolumn{4}{|c|}{$\begin{array}{l}\text { Meta-analysis (cases=3139, } \\
\text { controls=11 078) }\end{array}$} \\
\hline & & & & & RAF & p Value & OR & p Value & OR & $p$ Value & OR & 12 & $\mathbf{Q}$ \\
\hline rs2476601 & 1 & 114377568 & PTPN22 & $A / G$ & 0.10 & $1.29 \mathrm{E}-05$ & 1.28 & $1.28 \mathrm{E}-05$ & 1.44 & $1.49 \mathrm{E}-09$ & 1.32 & 0 & 0.65 \\
\hline rs4795067 & 17 & 26106675 & NOS2 & $\mathrm{G} / \mathrm{A}$ & 0.34 & $1.94 \mathrm{E}-07$ & 1.21 & $7.42 \mathrm{E}-03$ & 1.25 & $5.27 \mathrm{E}-09$ & 1.22 & 0 & 0.75 \\
\hline rs984971 & 2 & 163224521 & KCNH7 & $\mathrm{G} / \mathrm{A}$ & 0.36 & $3.62 \mathrm{E}-06$ & 0.84 & 0.02 & 0.87 & $2.29 \mathrm{E}-07$ & 0.85 & 0 & 0.61 \\
\hline rs1306395 & 2 & 61076272 & LINC01185 & $\mathrm{C} / \mathrm{T}$ & 0.43 & $2.99 \mathrm{E}-05$ & 0.86 & 0.04 & 0.88 & $3.43 \mathrm{E}-06$ & 0.87 & 0 & 0.85 \\
\hline rs7552167 & 1 & 24518643 & IFNLR1 & $\mathrm{A} / \mathrm{G}$ & 0.14 & $1.53 \mathrm{E}-05$ & 0.79 & 0.10 & 0.88 & $7.36 \mathrm{E}-06$ & 0.82 & 35.6 & 0.20 \\
\hline rs8106664 & 19 & 10728030 & SLC44A2 & $\mathrm{G} / \mathrm{T}$ & 0.23 & $3.28 \mathrm{E}-06$ & 0.81 & 0.13 & 0.89 & $1.67 \mathrm{E}-06$ & 0.83 & 0 & 0.52 \\
\hline rs2392581 & 7 & 38573234 & AMPH & $\mathrm{G} / \mathrm{A}$ & 0.42 & $6.90 \mathrm{E}-05$ & 0.87 & 0.17 & 0.93 & 4.42E-05 & 0.88 & 51.7 & 0.10 \\
\hline rs8103241 & 19 & 13122612 & NFIX & $\mathrm{G} / \mathrm{A}$ & 0.46 & $9.08 \mathrm{E}-05$ & 0.87 & 0.19 & 0.92 & $5.41 \mathrm{E}-05$ & 0.88 & 0 & 0.52 \\
\hline rs1133071 & 9 & 32455674 & DDX58 & $\mathrm{C} / \mathrm{T}$ & 0.30 & $3.36 \mathrm{E}-05$ & 1.17 & 0.20 & 1.09 & $2.49 \mathrm{E}-05$ & 1.15 & 64.9 & 0.06 \\
\hline rs6713082 & 2 & 62516544 & B3GNT2 & $\mathrm{A} / \mathrm{C}$ & 0.24 & $4.59 \mathrm{E}-05$ & 1.18 & 0.46 & 1.05 & $9.44 \mathrm{E}-05$ & 1.15 & 71.7 & 0.03 \\
\hline rs2298428 & 22 & 21982892 & YDJC & $\mathrm{T} / \mathrm{C}$ & 0.18 & $4.38 \mathrm{E}-05$ & 1.20 & 0.56 & 1.04 & $2.35 \mathrm{E}-04$ & 1.14 & 66.8 & 0.03 \\
\hline rs8016947 & 14 & 35832666 & NFKBIA & $\mathrm{T} / \mathrm{G}$ & 0.44 & $9.65 \mathrm{E}-05$ & 0.87 & 0.73 & 1.02 & $1.49 \mathrm{E}-03$ & 0.91 & 70.1 & 0.04 \\
\hline rs7895120 & 10 & 129064193 & DOCK1 & $\mathrm{T} / \mathrm{C}$ & 0.14 & $5.29 \mathrm{E}-05$ & 0.80 & 0.87 & 1.01 & $1.44 \mathrm{E}-03$ & 0.87 & 62.9 & 0.04 \\
\hline
\end{tabular}

bp, base position; chr, chromosome; $I^{2}$, heterogeneity index for ORs; $Q$, Cochrane's $Q$ statistic for heterogeneity of ORs; RAF, risk allele frequency;

a significance threshold of $\mathrm{p}<1 \times 10-4 .^{7}$ Genotyping was performed using the Life Technologies TaqMan chemistry on the QuantStudio genotyping platform at the University of Erlangen, Germany. Sample and SNPs with low call rates $(<0.9)$ were excluded prior to analysis. All genotype cluster plots were manually reviewed and SNPs were screened for deviation from Hardy-Weinberg equilibrium in control samples (Bonferroni corrected $\mathrm{p}<3.3 \times 10^{-3}$ ).

\section{Statistical analysis}

Association testing was performed using logistic regression implemented in PLINK and meta-analysis of Immunochip and validation summary statistics was performed, weighting SNPs by inverse-variance and assuming fixed effects, using the software package METAL.

For loci not previously reported as being associated with psoriasis susceptibility we investigated PsA-specificity using two large psoriasis studies. First, we tested association to psoriasis using genotype data from WTCCC2 and association summary statistics from the largest psoriasis study to date, consisting of 10588 psoriasis cases and 22806 controls, ${ }^{8}$ from ImmunoBase (http:// www.immunobase.org). Second, we compared effect estimates in PsA to psoriasis using multinomial logistic regression using genotype data for PsA cases and controls from Immunochip and psoriasis genotype data from WTCCC2 performed in Stata. Finally, we directly compared PsA and psoriasis genotypes, with PsA coded as cases and psoriasis coded as controls. Sex differentiated associations were investigated by analysing men and women separately and comparing differences in effect estimates using Cochrane's Q statistic using Immunochip genotype data.

To control for phenotype misclassification with rheumatoid arthritis (RA), we included a genetic risk score (GRS) comprised of the 41 non-HLA RA susceptibility SNPs reported in the RA Immunochip study, weighted by odds ratio (OR), as a covariate and recalculated the PsA Immunochip summary statistics. ${ }^{9} 10$

\section{RESULTS}

Following quality control of the validation genotype data a total of 13 SNPs for 1177 cases and 2155 controls was available for analysis. Meta-analysis of the validation samples with
Figure 1 Regional association plots for the PTPN22 locus for PSA Immunochip data and meta-analysis of rs2476601. The $x$-axis represents chromosomal position and gene location. The first y-axis represents $\log _{10}$ of the observed $p$ value from logistic regression, secondary y-axis represents estimated recombination rates $(\mathrm{cM} / \mathrm{Mb})$. Circles represent genotyped single nucleotide polymorphisms (SNPs), colour of the circle represents linkage disequilibrium $\left(r^{2}\right)$ with the index SNP (purple circle). $\mathrm{kb}$, kilobase; $\mathrm{cM}$, centimorgan; $\mathrm{Mb}$, megabase.

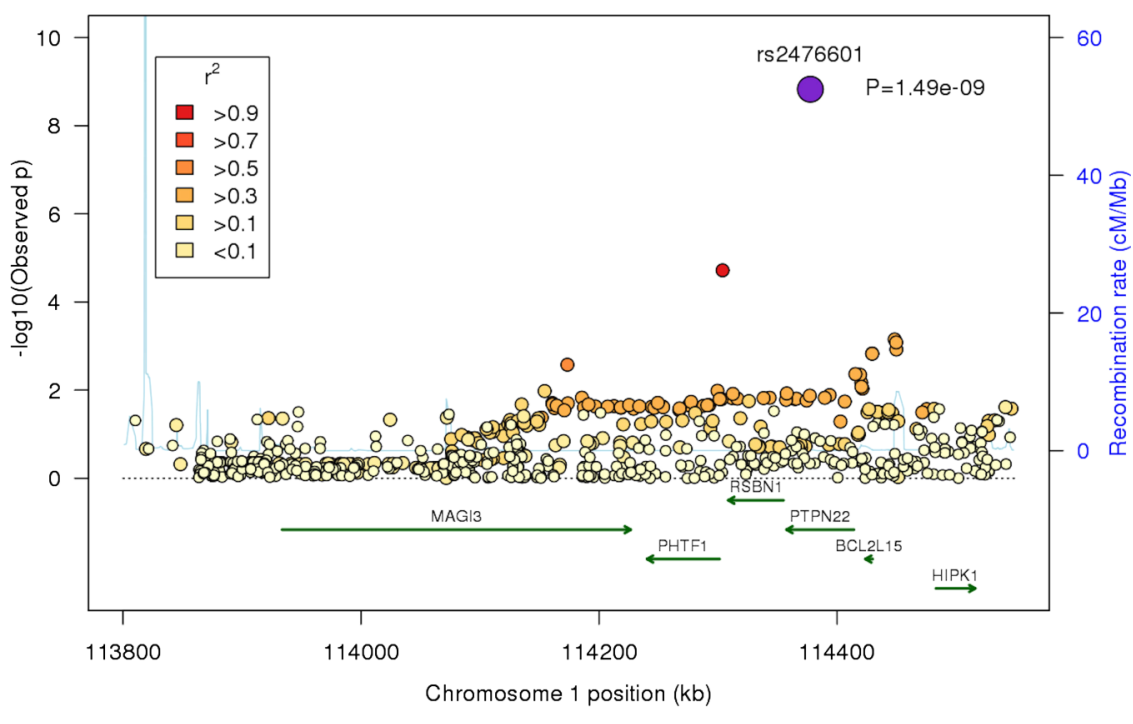


Figure 2 Forest plot of effect estimates for rs2476601 from the Immunochip, validation and meta-analysis. Rows are labelled by study group and include MAF, p values, ORs and $95 \%$ Cls. Reported MAF is estimated from control group, for Immunochip cohort this is estimated from UK controls. $\mathrm{Cl}$, confidence interval; minor allele frequency; OR, odds ratio.

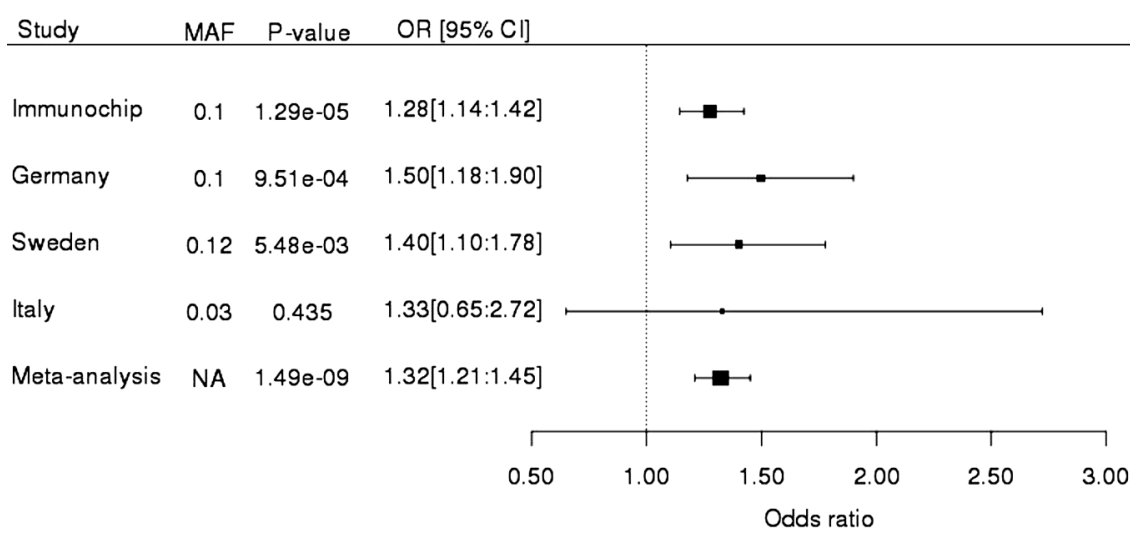

Immunochip data resulted in a combined data set of 3139 PsA cases and 11078 controls. We identified genome-wide significance to two loci; NOS2 (rs4795067, p =5.27 $\times 10^{-9}$ ) and PTPN22 (rs2476601, $\mathrm{p}=1.49 \times 10^{-9}$ ) (table 1). Association to NOS2 has previously been reported to psoriasis; however no such association has been made to PTPN22 (figures 1 and 2). Interestingly we observe a higher effect estimate for rs2476601 in men compared with women (1.31 vs 1.22 , respectively) as previously reported for this SNP in PsA, however this difference is not statistically significant $(\mathrm{Q}=0.52)$. We also observe a much lower minor allele frequency for rs 2476601 in the Italian population which is consistent with previous studies demonstrating a North-East to South-West gradient for minor allele frequency (MAF) across continental Europe. ${ }^{11}$

As SNPs at the PTPN22 locus have not previously been reported to be associated to psoriasis susceptibility we investigated this further in two large psoriasis data sets. First we analysed genotyped data from the WTCCC2 psoriasis study, excluding known PsA samples (cases $n=1784$, controls $n=5175)$, for rs2476601 and found no evidence for association $(p=0.34)$. Second we searched summary statistics from the largest psoriasis study to date (cases $n=10588$, controls $\mathrm{n}=22$ 806) using the ImmunoBase database and again found no evidence for association of rs2476601 to psoriasis susceptibility $(p=0.49)$. Using genotype data from the PsA Immunochip study and WTCCC2 we directly compared the effect estimates for rs2476601 in PsA and psoriasis using multinomial logistic regression and we found the estimates to be significantly different $\left(p=3.2 \times 10^{-4}\right)$. A direct comparison of genotypes for PsA $(n=1962)$ and psoriasis $(n=1784)$ found significant association to an increased risk of PsA $\left(\mathrm{p}=4.4 \times 10^{-4}, \mathrm{OR}=1.3\right)$.

Given that rs 2476601 is a genetic risk factor for RA we were concerned that the observed $\mathrm{p}$ value in the discovery study was a false positive due to phenotype misclassification caused by the presence of unidentified RA samples in the case cohort. However, we found the association to rs22476601 in the PsA Immunochip data was unaffected by the inclusion of the RA-GRS $\left(\mathrm{p}=1.29 \times 10^{-5}\right.$ vs $\left.\mathrm{P}_{\mathrm{GRS}}=1.30 \times 10^{-5}\right)$.

\section{DISCUSSION}

In this study we present evidence for association of rs 2476601 to susceptibility of PsA exceeding the threshold recognised as genome-wide significant $\left(\mathrm{p}<5 \times 10^{-8}\right)$ for the first time. In addition we used genotype data and summary statistics from two large psoriasis studies to demonstrate that this locus is differentially associated to PsA and not psoriasis per se. We also confirm association of PsA with a previously reported psoriasis locus, NOS2, bringing the total number of confirmed, genome-wide significant, PsA loci to 10 including 4 that are PsA-specific (HLA-B, chromosome 5q31, PsA-specific variants within IL23R and now PTPN22). Studies have shown that PTPN22 is a potent inhibitor of $\mathrm{T}$ cell activation and it is possible that the effect may differ between $\mathrm{T}$ cell subpopulations. ${ }^{12}$ For example we have shown that CD8 + T cells are important for PsA, while this has not been reported in psoriasis. ${ }^{5}$

Strengths of the current study include the large sample sizes used, which allowed us to confirm association at accepted genome-wide thresholds. Previous studies of this locus in PsA have been limited by small sample size; results have either shown weak evidence for association; ${ }^{13}{ }^{14}$ weak association in men only ${ }^{15}$ or no evidence for association at all. ${ }^{16}$ Indeed, our previous attempts to investigate rs2476601 and PsA susceptibility failed to find any evidence of association. ${ }^{17}$ This previous study had approximately $60 \%$ power to detect an effect of the size estimated in the current study. The absence of association for rs2476601 in the Italian cohort of this study is attributed to reduced power due to the much lower MAF (figure 2). Previous investigations of the rs2476601 PTPN22 variant with psoriasis have consistently reported no evidence for association, ${ }^{18}{ }^{19}$ but some have found association to other variants in the region, for example to rs3789604 (RSBN1) or haplotypes spanning PTPN22. ${ }^{20} 21$ However, in the largest psoriasis genetic associeither rs2476601 or $r 33789604(p=0.49$ and $p=1.00$, respectively). ${ }^{8}$ Indeed, a direct comparison of psoriasis and PsA confirmed that the rs2476601 association is PsA-specific, making it the fourth such locus to be identified.

In contrast to the previous reports, the study presented here is performed in a large cohort of 3139 cases and 11078 controls, includes independent validation and, for the first time, reports confirmed association with susceptibility to PsA exceeding genome-wide significance $\left(p=1.49 \times 10^{-9}\right)$. The identification of PsA-specific loci is vital in terms of understanding the different pathways involved, which may require different treatments, and for future screening strategies to identify subjects at risk of developing PsA in patients with psoriasis.

The SNP, rs2476601, has been found to be associated with multiple autoimmune diseases including RA, where the association is predominantly found in anti-citrullinated protein antibody (ACPA)-positive subjects, although association in the ACPA-negative subgroup has been reported. ${ }^{22}$ One possibility, therefore, is that the association with PsA could be due to the inclusion of patients with RA and coincidental psoriasis in the PsA cohort. Unfortunately, ACPA or rheumatoid factor status was not available for many samples. A strength of the current study, however, is that we used a GRS of known RA loci, which ation study performed to date, no association was detected to 
has been previously shown to adequately control for potential phenotype misclassification, to explore this possible confounder and found that the association with PsA remained statistically significant even after this adjustment. ${ }^{10}$

In conclusion we report for the first time genome-wide significant association of the rs2476601 variant in the PTPN22 gene with susceptibility to PsA consistent with reports in many other autoimmune diseases. In addition, we use genotype data from a large psoriasis study to demonstrate that rs2476601 is differentially associated to PsA and not psoriasis.

\section{Author affiliations}

${ }^{1}$ Arthritis Research UK Centre for Epidemiology, Centre for Musculoskeletal Research, Institute for Inflammation and Repair, Manchester Academic Health Science Centre, The University of Manchester, Manchester, UK

${ }^{2}$ Institute of Human Genetics, University of Erlangen-Nuremberg, Erlangen, Germany ${ }^{3} \mathrm{NIHR}$ Manchester Musculoskeletal Biomedical Research Unit, Central Manchester University Hospitals NHS Foundation Trust, Manchester Academic Health Science Centre, Manchester, UK

${ }^{4}$ The Kellgren Centre for Rheumatology, Central Manchester Foundation Trust, NIHR Manchester Biomedical Research Centre, Manchester, UK

${ }^{5}$ Monash University, Melbourne, Victoria, Australia

${ }^{6}$ NIHR-Leeds Musculoskeletal Biomedical Research Unit, Leeds Institute of Molecular Medicine, University of Leeds, Leeds, UK

${ }^{7}$ Department of Clinical Medicine, Institute of Molecular Medicine, Trinity College Dublin, Dublin, Ireland

${ }^{8}$ Adelaide and Meath Hospital and Trinity College Dublin, Dublin, Ireland

${ }^{9}$ Royal National Hospital for Rheumatic Diseases and Department Pharmacy and Pharmacology, University of Bath, Bath, UK

${ }^{10}$ Department of Public Health and Clinical Medicine, Rheumatology, University Hospital, Umeå, Sweden

${ }^{11}$ Department of Biopathology, Centre of Excellence for Genomic Risk Assessment in Multifactorial and Complex Diseases, School of Medicine, University of Rome 'Tor Vergata' and Fondazione PTV 'Policlinico Tor Vergata', Rome, Italy

${ }^{12}$ Rheumatology Department, Haywood Hospital, Health Services Research Unit, Institute of Science and Technology in Medicine, Keele University

${ }^{13}$ Department of Rheumatology, St. Vincent's University Hospital, UCD School of Medicine and Medical Sciences and Conway Institute of Biomolecular and Biomedical Research, University College Dublin, Dublin, Ireland

${ }^{14}$ The University of Queensland Diamantina Institute, Translational Research Institute, Princess Alexandra Hospital, Woolloongabba, Brisbane, Queensland, Australia ${ }^{15}$ Division of Rheumatology and Fraunhofer IME-Project-Group Translational Medicine and Pharmacology, Goethe University, Frankfurt, Germany

Acknowledgements The authors acknowledge the assistance given by IT Services and the use of the Computational Shared Facility (CSF) at The University of Manchester. The authors thank Arthritis Research UK for their support (grant ref 20385) and the NIHR Manchester Musculoskeletal Biomedical Research Unit. This report includes independent research funded by the National Institute for Health Research Biomedical Research Unit Funding Scheme. The views expressed in this publication are those of the author(s) and not necessarily those of the NHS, the National Institute for Health Research or the Department of Health. The authors gratefully acknowledge the contribution of patients and staff of the Early Swedish Psoriatic Arthritis Registry (SwePsA).

Contributors $A B$ devised the study concept and design. JB performed statistical analysis. JB and $A B$ wrote the manuscript. $S L$ and $U H$ performed validation genotyping and contributed to statistical analysis. $A B-A$ and $S U$ contributed to the statistical analysis. $F B, H B$ and $A R$ contributed to interpretation of findings. INB, HM-O, PHe, AWR, DK, EK, G-MA, EG, JP, RM, OF, NM, PHo, MAB and MF contributed data to the discovery phase. All authors contributed to and approved the manuscript.

Funding Frankfurt: the German Federal Ministry of Education and Research ArthroMark (project 4, 01 EC 1009C), the Federal State of Hesse (LOEWE-project: IME Fraunhofer Project Group Translational Medicine \& Pharmacology at the Goethe University), HB received funding from Pfizer Pharma, Germany (Forschungsförderpreis Rheumatologie 2012). Support for the Australian component of the study was received from Abbvie. MAB is funded by a National Health and Medical Research Foundation (Australia) Senior Principal Research Fellowship.

Competing interests None declared.
Ethics approval All samples were collected with approval from the respective local ethical committee: the medical faculties of the Universities of Erlangen and Münster, the University of Tor Vergata of Rome and the Umeå University, Sweden.

Provenance and peer review Not commissioned; externally peer reviewed.

Data sharing statement All summary statistics for data generated in this study are presented in table 1. Further information can be obtained by contacting the authors.

Open Access This is an Open Access article distributed in accordance with the terms of the Creative Commons Attribution (CC BY 4.0) license, which permits others to distribute, remix, adapt and build upon this work, for commercial use, provided the original work is properly cited. See: http://creativecommons.org/ licenses/by/4.0/

\section{REFERENCES}

1 Ibrahim G, Waxman R, Helliwell PS. The prevalence of psoriatic arthritis in people with psoriasis. Arthritis Rheum 2009:61:1373-8.

2 Rosen CF, Mussani F, Chandran V, et al. Patients with psoriatic arthritis have worse quality of life than those with psoriasis alone. Rheumatology (Oxford) 2012;51:571-6.

3 Karason A, Love TJ, Gudbjornsson B. A strong heritability of psoriatic arthritis over four generations - the Reykjavik Psoriatic Arthritis Study. Rheumatology (Oxford) 2009;48:1424-8.

4 Okada Y, Han B, Tsoi LC, et al. Fine mapping major histocompatibility complex associations in psoriasis and its clinical subtypes. Am J Hum Genet 2014;95:162-72. http://www.ncbi.nlm.nih.gov/pubmed/25087609 (accessed 15 Sep 2014).

5 Bowes J, Budu-Aggrey A, Huffmeier U, et al. Dense genotyping of immune-related susceptibility loci reveals new insights into the genetics of psoriatic arthritis. Nat Commun 2015:6:6046

6 Strange A, Capon F, Spencer CCA, et al. A genome-wide association study identifies new psoriasis susceptibility loci and an interaction between HLA-C and ERAP1. Nat Genet 2010;42:985-90.

7 Smyth DJ, Plagnol V, Walker NM, et al. Shared and distinct genetic variants in type 1 diabetes and celiac disease. N Engl J Med 2008;359:2767-77.

8 Tsoi LC, Spain SL, Knight J, et al. Identification of 15 new psoriasis susceptibility loci highlights the role of innate immunity. Nat Genet 2012;44:1341-8.

9 Eyre $S$, Bowes J, Diogo D, et al. High-density genetic mapping identifies new susceptibility loci for rheumatoid arthritis. Nat Genet 2012;44:1336-40.

10 Han B, Diogo D, Eyre $S$, et al. Fine mapping seronegative and seropositive rheumatoid arthritis to shared and distinct HLA alleles by adjusting for the effects of heterogeneity. Am J Hum Genet 2014;94:522-32.

11 Gregersen PK, Olsson LM. Recent advances in the genetics of autoimmune disease. Annu Rev Immunol 2009;27:363-91.

12 Stanford SM, Bottini N. PTPN22: the archetypal non-HLA autoimmunity gene. Nat Rev Rheumatol 2014;10:602-11.

13 Butt C, Peddle L, Greenwood C, et al. Association of functional variants of PTPN22 and tp53 in psoriatic arthritis: a case-control study. Arthritis Res Ther 2006:8:R27.

14 Juneblad K, Johansson M, Rantapää-Dahlqvist S, et al. Association between the PTPN22 +1858 C/T polymorphism and psoriatic arthritis. Arthritis Res Ther 2011;13:R45.

15 Hüffmeier $U$, Reis $A$, Steffens $M$, et al. Male restricted genetic association of variant R620W in PTPN22 with psoriatic arthritis. J Invest Dermatol 2006:126:932-5.

16 Hinks A, Barton A, John S, et al. Association between the PTPN22 gene and rheumatoid arthritis and juvenile idiopathic arthritis in a UK population: further support that PTPN22 is an autoimmunity gene. Arthritis Rheum 2005;52:1694-9.

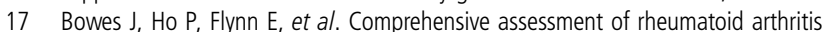
susceptibility loci in a large psoriatic arthritis cohort. Ann Rheum Dis 2012;71:1350-4

18 Zheng J, Ibrahim S, Petersen F, et al. Meta-analysis reveals an association of PTPN22 C1858T with autoimmune diseases, which depends on the localization of the affected tissue. Genes Immun 2012;13:641-52.

19 Nistor I, Nair RP, Stuart P, et al. Protein tyrosine phosphatase gene PTPN22 polymorphism in psoriasis: lack of evidence for association. J Invest Dermatol 2005; 125:395-6.

20 Smith RL, Warren RB, Eyre S, et al. Polymorphisms in the PTPN22 region are associated with psoriasis of early onset. Br J Dermatol 2008;158:962-8.

21 Hüffmeier $U$, Steffens $M$, Burkhardt $H$, et al. Evidence for susceptibility determinant (s) to psoriasis vulgaris in or near PTPN22 in German patients. J Med Genet 2006;43:517-22

22 Viatte S, Plant D, Bowes J, et al. Genetic markers of rheumatoid arthritis susceptibility in anti-citrullinated peptide antibody negative patients. Ann Rheum Dis 2012:71:1984-90. 